Astrid Köhler · Salonkultur im klassischen Weimar 


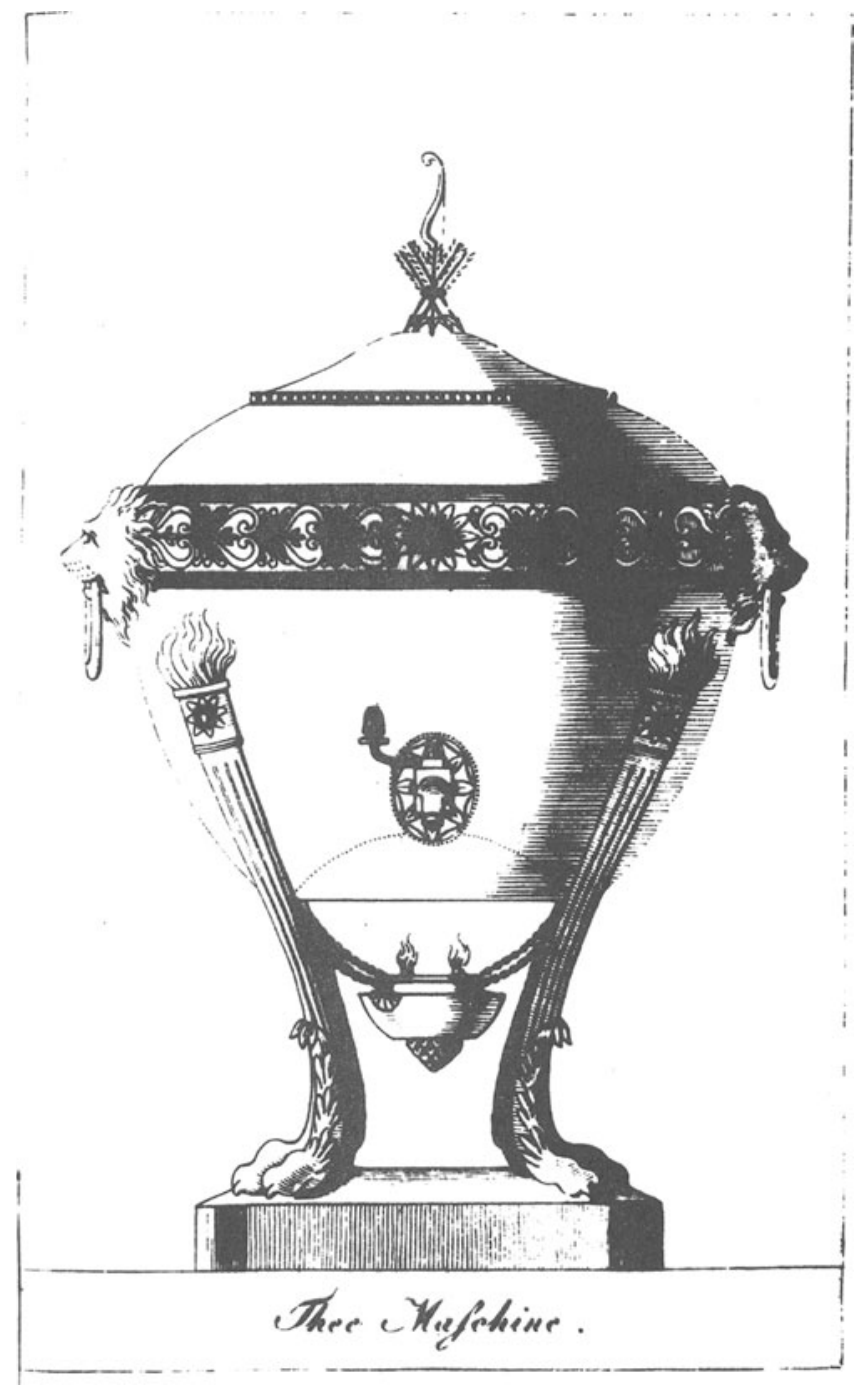

Abb. 1

Zeitgenössische Teemaschine 


\section{Astrid Köhler}

\section{Salonkultur im \\ klassischen Weimar}

Geselligkeit als Lebensform und literarisches Konzept 
Die Deutsche Bibliothek - CIP-Einheitsaufnahme

\section{Köhler, Astrid:}

Salonkultur im klassischen Weimar : Geselligkeit als

Lebensform und literarisches Konzept / Astrid Köhler.

- Stuttgart : M und P, Verl. für Wiss. und Forschung, 1996

Zugl.: Berlin, Freie Univ., Diss., 1994

ISBN 978-3-476-45145-3

ISBN 978-3-476-45145-3

ISBN 978-3-476-04241-5 (eBook)

DOI 10.1007/978-3-476-04241-5

Dieses Werk ist einschließlich aller seiner Teile geschützt. Jede Verwertung außerhalb der engen Grenzen des Urheberrechtsgesetzes ist ohne Zustimmung des Verlages unzulässig und strafbar. Das gilt insbesondere für Vervielfältigungen, Übersetzungen, Mikroverfilmungen und Einspeichenung in elektronischen Systemen.

M \& P Verlag für Wissenschaft und Forschung ein Verlag der J.B. Metzlerschen Verlagsbuchhandlung und Carl Emst Poeschel Verlag GmbH in Stuttgart

(c) 1996 Springer-Verlag GmbH Deutschland Ursprünglich erschienen bei J.B. Metzlersche Verlagsbuchhandlung und Carl Ernst Poeschel Verlag GmbH in Stuttgart 1996 


\section{Vorwort}

Das vorliegende Buch ist eine leicht überarbeitete Fassung meiner Dissertation, die im Sommer 1994 am Fachbereich Germanistik der Freien Universität Berlin angenommen wurde. Die Arbeit ist finanziell durch die Friedrich-Schiller-Universität Jena und die Hans-Böckler Stiftung in Düsseldorf gefördert worden. Letzterer sowie dem Gonville \& Caius College Cambridge habe ich auch großzügige Beihilfen zum Druckkostenzuschuß für das Buch zu danken. Der Charakter der Dissertation brachte lange Tage und Wochen an Archivarbeit mit sich; besonders danke ich den Mitarbeitern des Goethe-und-Schiller-Archivs in Weimar, des Schopenhauer-Archivs in Frankfurt und des Goethe-Museums in Düsseldorf für ihre tätige Hilfe.

Die Freunde in Jena, Berlin und anderswo haben mir durch kritische Lektüre und Kommentare und ihre ganz eigene Teilnahme an meinem Gegenstand geholfen: eine gegenwärtige Form von Salonkultur. Meiner Familie und meinem Partner Günter Regneri habe ich moralische und organisatorische Unterstützung und die oft genug nötige Aufmunterung zur Arbeit zu verdanken, Heike Hauß opferte viel Zeit für die typographische Gestaltung des Texts.

Mein größter Dank aber geht an meine Mentorin Frau Dr. Claudia Albert, die die Entstehung der Arbeit nach meinem Wechsel nach Berlin mit freundlicher Geduld, stets aufmerksamer Lektüre und wertvollen Hinweisen begleitet hat. 


\section{INHALTSVERZEICHNIS}

I.

EINLEITUNG: DER TEETISCH

I.1. Die Initiationssituation 14

I.2. Die Wirtin: Johanna Schopenhauer 15

I.3. Die Stadt: Weimar 20

I.4. Voraussetzungen, Intentionen, Effekte 24

II. ZUGÄNGE ZUR GESELLIGKEIT 29

II.1. Das Quellenkorpus: Selektion als historisches Prinzip 29

II.1.1. Ästhetische Kanonisierung 29

II.1.2. Biographische und familienhistorische Auslese $\quad 32$

II.1.3. Kulturpolitische Tendenzen 37

II.1.4. Wissenschaftshistorische und -politische Selektionen 39

II.2. Der Forschungsstand zum Schwerpunkt Geselligkeit

II.3. Wissenschaftliches Interesse und methodisches Vorgehen 54

II.3.1. Strukturen in Bewegung 54

II.3.2. Die Autoreninstanzen und der Charakter des Textmaterials

III. GESELLIGE PRAXIS - STRUKTUREN KULTURELLER UND SOZIALER ORDNUNG IM SALON 65

III.1. Raum- und Zeitstrukturen der Geselligkeit $\quad 65$ 
III.1.1. 1806-1813 $\quad 65$

III.1.2. 1814-1824 78

III.1.3. 1824-1829 $\quad 83$

III.2. Mitgliederstrukturen 91

III.3. Strukturen geselliger Praxis 98

III.3.1. Geselliges Geschehen 100

III.3.1.1. Die Arbeit am Ofenschirm 101

III.3.1.2. Der Gesangsabend (31.12.1807) 107

III.3.2. Kommunikationsstrukturen im Salon:

Eine Exkommunikation aus der Geselligkeit Gruppenkonsens und Außenseiter 111

$\begin{array}{lll}\text { III.3.3. } & \text { Salon und Residenz: } \\ & \text { Der Maskenzug vom 3. Februar } 1809 & 120\end{array}$

III.3.4. Synopse 130

IV. GESELLIGE FIKTION - ZUR KONSTITUTION UND AUFLÖSUNG SOZIALER ORDNUNG IN DEN ROMANEN

IV.1. Die Wahlverwandtschaften (1809) 137

IV.1.1. Raum- und Zeitstrukturen 139

$\begin{array}{lll}\text { IV.1.2. } & \text { Figurationen I: Muster, vor- und } \\ \text { nachgezeichnet } & 147\end{array}$

IV.1.3. Figurationen II: Musterüberschreitung 149

$\begin{array}{lll}\text { IV.1.4. } & \text { Außerhalb der Geselligkeit: } & \\ & \text { Angrenzende Figuren/gruppen } & 150\end{array}$

$\begin{array}{ll}\text { IV.1.5. Die Sozialstruktur der } & \\ & \text { wahlverwandtschaftlichen Gruppe }\end{array}$

IV.1.6. Das Agieren der Gruppe auf dem Landgut $\quad 155$

IV.1.6.1. Der Umgang mit der natürlichen und gebauten Umgebung 157

IV.1.6.2. Der Umgang mit der Dorfbevölkerung 159 
IV.1.7. Die Kommunikationsstrukturen in der

$\begin{array}{lll} & \text { Gruppe } & 162 \\ \text { IV.1.7.1. } & \text { Das Eröffnungsgespräch } & 164\end{array}$

IV.1.7.2. Das Gleichnisgespräch 167

IV.1.7.3. Ottilies Erscheinen 169

IV.1.7.4. Die Reaktionen auf den Kindestod 174

IV.1.7.5. Ottilies Bekenntnismonolog 175

IV.1.8. Ottilies Sonderposition in der Gruppe 178

IV.1.9. Synopse 181

IV.2. Gabriele (1819) 184

IV.2.1. Johanna Schopenhauer als Romanautorin 184

IV.2.2. Abriß der äußeren Romanhandlung 187

$\begin{array}{lll}\text { IV.2.3. } & \text { Raum- und Zeitstrukturen } & 189\end{array}$

$\begin{array}{lll}\text { IV.2.4. Figurationen } & 194\end{array}$

IV.2.5. Außerhalb der Geselligkeit: Angrenzende Figuren/gruppen 197

IV.2.6. Die Sozialstruktur der Gruppe 199

$\begin{array}{lll}\text { IV.2.7. Strukturen der Kommunikation } & 202\end{array}$

IV.2.8. Die Funktion der Kunst für die Gruppe

$\begin{array}{lll}\text { IV.2.9. Synopse } & 213\end{array}$

IV.3. Gabriele und die Wahlverwandtschaften -

IV.3.1. $\quad$ Gruppenstrukturen und extern orientierte Ordnung 219

IV.3.2. Gesellige Praktiken 220

IV.3.3. Gabriele und Ottilie: Charakter und

Funktion der Heldinnen

V. GESELLIGKEIT ALS SOZIALES

EXPERIMENTTIERFELD: MACHTSPIELE AM

TEETISCH. SCHLUSS.

V.1. Entstehung und Funktion der sozialen

Binnenordnung im Salon 
V.2. Die Analysen zur Geselligkeitskultur und ihre Ergebnisse

V.3. Salonkultur und Romankultur soziale Praxis und soziale Fiktion

VI. LITERATURVERZEICHNIS

VI.1.

Primärliteratur

VI.2.

Forschungsliteratur

VI.3. Veröffentlichte Quellen

VI.3.1. Kataloge, Stadtführer und -geschichten,

Überblicksdarstellungen zum klassischen

Weimar

VI.3.2. Personenporträts, Erinnerungsschriften, Detaildarstellungen

VI.3.3. Tagebücher, Briefe, Gespräche, Zeichnungen

VI.3.4.

Zeitgenössische Zeitschriften

VI.4.

Unveröffentlichte Quellen:

VI.4.1.

Briefe an Johanna Schopenhauer

256

VI.4.2.

Briefe von Johanna Schopenhauer

257

VI.4.3.

Andere Autographen

264

VI.5.

Verzeichnis der Abbildungen 